Revista Científica de FAREM-Estelí

Medio ambiente. tecnología y desarrollo humano

Año 10 | Edición especial: artículos de revisión documental

ISSN: 2305-5790

https://rcientificaesteli.unan.edu.ni

DOI: https://doi.org/10.5377/farem.v0i0.11607

\section{Entornos Virtuales de Aprendizaje (EVA) para la enseñanza- aprendizaje de la Matemática}

\section{Virtual learning environment (VLEs) for the teaching-learning of Mathematics}

\section{José Ismael González}

Docente de la escuela secundaria Reino de Suecia. Estelí, Nicaragua. Ministerio de Educación de Nicaragua (MINED). Estudiante de doctorado en Gestión y Calidad de la Investigación Científica II Cohorte 2020-2023 (UNAN-Managua)

https://orcid.org/0000-0001-5232-0266

joseismael26@yahoo.com

\section{Julia Granera}

Doctora en Matemática Aplicada. Docente titular. FAREM-Estelí, UNAN-Managua. igranerar@gmail.com

\section{RESUMEN}

El objetivo del presente estudio fue analizar los Entornos Virtuales de Aprendizaje (EVA) y su aplicación en la asignatura de Matemática. Para ello, se realizó una revisión de investigaciones relacionadas con la temática. En cuanto a lo metodológico, se hizo un análisis de contenido, mediante revisión documental-bibliográfico de estudios publicados entre 2010 - 2020. Para la selección del material objeto de análisis se tomó como criterio temático, pertinencia, relevancia, idioma, año de publicación y lugar de procedencia. En relación a las fuentes de información, se utilizó las bases de datos de la UNAN-Managua EBSCO, RIUMA, E-Libro, también información académica de fuentes confiables, localizadas mediante el buscador Google académico. A partir de la búsqueda efectuada se realizó un análisis bibliométrico y contenido cualitativo, para seleccionar las publicaciones más relevantes con los intereses de este estudio. Los resultados más relevantes muestran los EVA como herramientas valiosas que ofrecen múltiples posibilidades para el desarrollo de enseñanza-aprendizaje acorde con las demandas educativas de hoy en día. Se concluye que los EVA, propician nuevas y distintas formas de enseñar y de aprender, en cualquier nivel del sistema educativo, como apoyo para lograr una educación de calidad.
RECIBIDO

$26 / 03 / 2021$

ACEPTADO

19/052021

\section{PALABRAS CLAVE}

Ambientes virtuales; aprendizaje; Matemática; estudiantes. 


\section{ABSTRACT}

The objective of this study was to analyze the Virtual Learning Environments (VLE) and their application in the Subject of Mathematic, for this purpose, a review of research related to the subject was carried out. In terms of methodology, a content analysis was carried out, by a documentary-bibliographic review of studies published between 2010-2020. The selection of the material to be analyzed, was based on criteria such as subject matter, pertinence, relevance, language, year of publication and place of origin. In relation to the sources of information, the UNAN-Managua databases EBSCO, RIUMA, E-Libro were used, as well as academic information from reliable sources, located through the Google Academic search engine. A bibliometric and qualitative content analysis was carried out from the search performed to select the most relevant publications with the interest of this study. The most relevant results show VLE as valuable tools that offer multiple possibilities for the development of teaching-learning according to today's educational demand. It is concluded that VLEs, provide new and different ways of teaching and learning, at any level of the educational system, as a support to achieve quality education.

\section{KEYWORDS}

Virtual Environments; learning; Mathematics; students. 


\section{INTRODUCCIÓN}

Las aulas virtuales son un factor de vital importancia en la transformación de diversos campos de la sociedad. Las herramientas tecnológicas, tienen el potencial de transformar la naturaleza de la educación en cuanto a dónde y cómo se produce el proceso de enseñanza-aprendizaje, así como de introducir cambios en los roles de los profesores y los estudiantes, y en las diferentes acciones que se realiza en el proceso educativo.

Las tecnologías de información y comunicación (TIC) en el ámbito educativo ha generado nuevas maneras de concebir y dirigir los procesos de enseñanza -aprendizaje. Estas tecnologías han sentado diversas posturas en cuanto a su incorporación e integración curricular y a las ventajas o desventajas que podrían tener en el desempeño académico de los estudiantes. Una de las áreas en las que han irrumpido con mayor fuerza es matemática, pues con la creación de diferentes software matemáticos como GeoGebra y Descartes, entre otros ha surgido un interés creciente por diseñar ambientes de aprendizaje (Córdoba, 2014).

Al incorporar el uso de tecnologías en contenidos matemáticos, permitirá tanto a los estudiantes como a los docentes expandir sus conocimientos, explorar sus capacidades y mejorar las habilidades en la resolución de ejercicios y/o problemas. Por esta razón, diversos autores han desarrollado estudios sobre la incorporación e impacto de las (TIC), en los procesos de enseñanza-aprendizaje.

Revelo \& Carrillo (2018) desarrollaron la investigación, "impacto del uso de las TIC como herramientas para el aprendizaje de la matemática de los estudiantes de educación media" en sus hallazgos concluye que los estudiantes logran una mejor comprensión con el uso de las TIC, considerando que, las innovaciones en la enseñanza de la Matemática son más efectivas si están incorporadas las tecnologías en los procesos pedagógicos que generen aprendizaje significativo entre estudiantes.

Por otra parte Coca (2013), realizó la investigación “Enseñanza-aprendizaje de la Matemática en Educación Secundaria Obligatoria (ESO)" según resultados de su estudio concluye que es fundamental un buen equipamiento tecnológico en las aulas para la enseñanza y el aprendizaje de la matemática, así como animar a los docentes a utilizar los recursos de las TIC y procedimientos que favorezcan la enseñanza de la Matemática.

Las TIC nos ofrecen diversidad de recursos de apoyo para los procesos de enseñanza como son: material didáctico, softwares interactivos, entornos virtuales, internet, blogs, wikis, webquest, foros, chat, mensajerías, videos conferencias y otros canales de comunicación y manejo de información. Estos recursos facilitan el desarrollo de la creatividad, innovación, entornos de

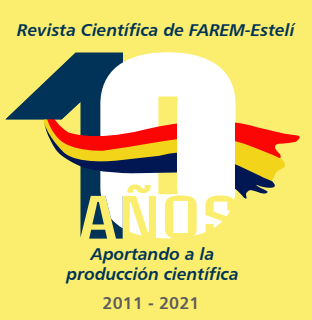


trabajo colaborativo, promoción del aprendizaje significativo, activo y flexible. (Rodriguez et al., 2017 p. 6)

A pesar de todo lo expuesto según los estudios realizados y considerando que la Matemática es una asignatura fundamental contemplada en todos los programas de estudio. En la mayoría de los casos se está desarrollando los contenidos de forma tradicional, considerándose estos métodos como modos de enseñar y aprender de manera repetitiva, memorística y poco significativo, generándose desinterés en los estudiantes, por tanto, bajo rendimiento.

Si bien, en los últimos años, las TIC han pasado a formar parte de nuestra vida cotidiana y constituyen una herramienta facilitadora de los procesos de enseñanza-aprendizaje de la Matemática, su uso en el aula requiere una metodología adecuada, (Rugama, 2017). En la actualidad, en virtud de los avances científicos y con ella los adelantos en materia educativa, se ha venido instando en el uso de las TIC como estrategias para adecuar la educación a los nuevos requerimientos que demanda la formación del estudiantado.

Tales exigencias requieren de docentes preparados en el uso y manejo de las TIC y como parte de ellas están los EVA, que facilitan espacios educativos a partir de un conjunto de herramientas informáticas que posibilitan la interacción didáctica. En este caso particular, los EVA, se constituyen en un ámbito propicio para la enseñanza aprendizaje de la matemática, al ser un recurso de trabajo básico en el aula de clases, que puede ser empleado tanto por docentes como por estudiantes y, gracias a herramientas como Internet la información está al alcance de todos.

"Un entorno virtual de aprendizaje es un espacio educativo alojado en la web, conformado por un conjunto de herramientas informáticas que posibilitan la interacción didáctica" (Gutiérrez Rodríguez, 2018, p. 281). De acuerdo con esta definición, un entorno virtual de aprendizaje (EVA) posee cuatro características básicas:

- Es un ambiente electrónico, no material en sentido físico, creado y constituido por tecnologías digitales.

- Está hospedado en la red y se puede tener acceso remoto a sus contenidos a través de algún tipo de dispositivo con conexión a Internet.

- Las aplicaciones o programas informáticos que lo conforman sirven de soporte para las actividades formativas de docentes y alumnos.

- La relación didáctica no se produce en ellos "cara a cara" (como en la enseñanza presencial), sino mediada por tecnologías digitales. Por ello los EVA permiten el desarrollo de acciones educativas sin necesidad de que docentes y alumnos coincidan en el espacio o en el tiempo.

La definición de estos entornos indica que presentan una dimensión tecnológica y una dimensión educativa, las cuales se interrelacionan y potencian entre sí. Los EVA diseñados, contienen una serie de actividades muy prácticas, que

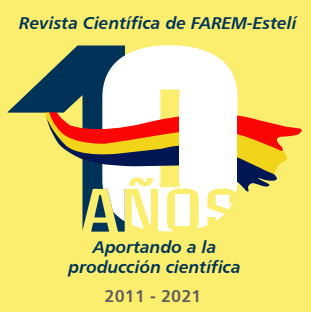


poseen un lenguaje sencillo, lo que facilita al estudiante la comprensión de los contenidos. Tiene recursos lúdicos y activos, de tal manera que el estudiante por iniciativa propia quiera hacer las actividades sin presión alguna y se sienta orgulloso de tener buenos resultados en sus pruebas o se anime frecuentemente a superar las dificultades que pueda encontrar, adquiriendo confianza y seguridad en su aprendizaje, y será capaz de enfrentar nuevas situaciones sin temor al error, lo que al final será de gran satisfacción para él, al alcanzar buenos resultados (Pino \& Salazar, 2018).

En la actualidad el acceso al contenido es más flexible y no se restringe a las paredes de un aula, las posibilidades de acceder a la información desde cualquier lugar que posea conexión a internet; combina distintos recursos para mejorar el proceso de enseñanza; facilitando el aprendizaje colaborativo y cooperativo, aumentando la motivación y participación de los estudiantes.

Por ello, el objetivo del presente estudio fue analizar los Entornos Virtuales de Aprendizaje (EVA) y su aplicación en la asignatura de Matemática. Considerando que la incorporación de nuevas estrategias didácticas mediante un EVA, son necesarias para ejercer la función docente en esta sociedad de la era digital.

De esta manera, actualmente, los procesos educativos virtuales están evolucionando y transformándose, por lo que en ocasiones es difícil conceptualizarlos y precisar su abordaje. Sin embargo, existen muchos autores que han dedicado gran parte de su tiempo a estudiarlos y conceptualizarlos.

A partir de lo expuesto, se reconoce que en los procesos de aprendizaje actuales los ambientes virtuales resultan ser el complemento perfecto para una educación presencial. En función de las premisas precedentes el problema que aborda el presente estudio, puede plantearse a través de la siguiente interrogante: ¿̇De qué manera un aula virtual, bajo la modalidad de un EVA, servirá como apoyo al aprendizaje de la Matemática?

A partir de la problemática es necesario proponer la implementación de estrategias didáctica utilizando un EVA para la enseñanza-aprendizaje de la Matemática. 


\section{MATERIALES Y MÉTODOS}

La realización de este artículo de revisión se sustentó en el paradigma cualitativo, desde un enfoque interpretativo, dado su orientación hacia un análisis de contenido, bajo la metodología de carácter documental bibliográfica. Como estrategia de búsqueda de información, se procedió a identificar, seleccionar y clasificar las fuentes de acuerdo a la temática, año de publicación y relevancia, en publicaciones que datan entre 2010 y 2020. Estas relacionadas al tema de los EVA, de literatura en español, en bases de datos de la UNAN-Managua, EBSCO, RIUMA y E-Libro. También, fueron incorporadas en este estudio investigaciones tomadas de otras fuentes de internet localizadas a través de Google académico, que se consideraron valiosas por sus aportes a la investigación.

La variable estudiada fue la producción de literatura científica relacionada con los EVA como estrategia para el aprendizaje de la Matemática. Para la recolección de las fuentes bibliográficas, se aplicó el modelo búsqueda a través de las palabras clave, todas relacionadas con los términos: entornos virtuales de aprendizaje, enseñanza de la matemática, educación, estrategias de enseñanza aprendizaje.

Una vez recopiladas un número importante de publicaciones que se ajustaron a las variables en estudio, objetivos de la investigación y los criterios establecidos. El análisis del estudio bibliométrico permitió seleccionar las publicaciones científicas, cuyos aportes se consideraron los más valiosos y pertinentes para el desarrollo de este estudio.

\section{RESULTADOS}

Una vez realizado el análisis de la información recolectada, en relación con los EVA y sus aplicaciones en la asignatura de Matemática, se constató a partir de reflexiones que los EVA, contribuyen como apoyo para mejorar en gran medida el desarrollo de procesos de enseñanza-aprendizaje de la Matemática, ofreciendo muchas ventajas y pueden ser utilizados en cualquier nivel de educación, de acuerdo con los sistemas educativos actuales.

Las TIC también permiten una interacción sujeto-máquina y la adaptación de ésta a las características educativas y cognitivas de la persona. De esta forma, los estudiantes dejan de ser meros receptores pasivos de información pasando a ser procesadores activos y conscientes de la misma (Pacheco et al., 2017). 
En la actualidad, se hace uso de las TIC en muchos aspectos de nuestra vida cotidiana, además de utilizarse en los procesos de aprendizaje. De esta manera, cuando se hace referencia a un EVA, permite comprender que utilizamos las TIC a la hora de aprender en la red de forma autónoma, reconociendo la importancia que adquiere el momento social y tecnológico en el que incidimos al momento de reflexionar sobre los entornos de aprendizaje. En este estudio se ha analizado información existente en relación con entornos virtuales, basándose en diferentes actores, en relación a la temática a continuación se discute sobre los principales hallazgos de este estudio.

De los resultados obtenidos en este estudio, se asume que en la actualidad existe un conocimiento importante acerca de los EVA. Según la literatura se presentan como espacios, softwares o aplicaciones informáticas que están dotados de materiales didácticos y recursos tecnológicos donde la comunicación y la interacción son esenciales, dado que pretenden ser lo más parecido posible a los espacios reales y sus aplicaciones en los procesos de enseñanza-aprendizaje de la Matemática.

Lo antes mencionado, coincide con los resultados presentados por (Rodriguez et al., 2017), quienes refieren que las TIC nos ofrecen diversidad de recursos de apoyo para los procesos de enseñanza. Estos recursos facilitan el desarrollo de la creatividad, innovación, entornos de trabajo colaborativo, promoción del aprendizaje significativo, activo y flexible. En este sentido, las TIC, han surgido como una herramienta con gran potencial para la mediación pedagógica.

La presencia de la tecnología es un fenómeno ubicuo e irreversible en el mundo contemporáneo, y la escuela no puede desconocer esta realidad, si quiere formar a adolescentes y jóvenes que sean capaces de integrarse en esta nueva sociedad de modo pleno. De acuerdo con Gutiérrez Rodríguez (2018) considera que enseñar con ambientes virtuales o más ampliamente con tecnología, es una necesidad para promover la inclusión en la vida social, cultural, económica y laboral del siglo XXI, en la cual la tecnología tiene un lugar fundamental.

Por otra parte, las características principales de los EVA, de acuerdo con los estudios realizados se coincide en que facilitan la colaboración, interactividad, flexibilidad, estandarización, usabilidad y accesibilidad a la comunicación e información que conlleva a un autoaprendizaje e interconexión favoreciendo claramente los distintos momentos del proceso de enseñanza-aprendizaje.

Según, Araque et al., (2018) en la visión constructivista de los EVA, la concepción del estudiante es la de constructor de su aprendizaje, en el cual participa de forma activa. En este sentido, enseñar en un EVA ayuda a los estudiantes adquirir distintas capacidades vinculadas con el entorno cultural del mundo contemporáneo, como, por ejemplo: el manejo instrumental de

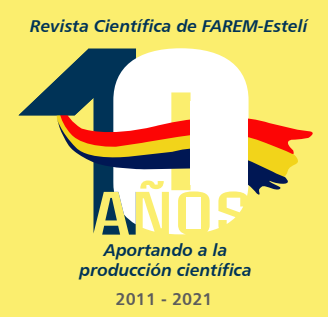


aplicaciones informáticas; la búsqueda y decodificación de la información hipertextual y multimedia allí publicada; la interacción con otros en un ambiente electrónico; la creación de contenidos digitales en distintos lenguajes, no sólo textual, sino también audiovisual.

Asimismo, Banega, (2009, p. 81) citado por, (Scharfstein \& Gaurf, 2013) en su tesis central considera los EVA, no solo como un texto, "sino que en ocasiones funcionan como contexto en que se realizan distintas prácticas mediadas por los sistemas semióticos multimodales." Lo anterior puede ser variado según las necesidades específicas. Esto tiene el propósito de ampliar las posibilidades de adaptar el objeto para varios cursos y contextos, a diferencia de un material que sea más extenso y abarque muchos contenidos.

Respecto al uso de EVA, se pueden utilizar principalmente como medio de interacción en el que se comparte información a la que se puede acceder en cualquier momento con la finalidad de mejorar el aprendizaje de los estudiantes y así mismo, contar con la oportunidad de evaluar los participantes.

Los Entornos Virtuales de Enseñanza y Aprendizaje son una fusión entre la tecnología y la pedagogía, específicamente a lo que subyace en ellos, las plataformas educativas son de suma importancia en los EVA ya que forman un espacio de interacción entre el profesor y el estudiante (Pineda \& Leyva, 2013).

En cuanto a los beneficios de los EVA en la asignatura de Matemática, este estudio los relaciona con la mejora de la calidad educativa, ya que complementa la educación presencial. Se incrementa el aprendizaje y la motivación de los estudiantes, al contar con mayor flexibilidad e independencia y contando con más tiempo disponible en los estudios. De esta manera se logra, aprender, trabajar de forma colaborativa, manteniéndose en comunicación con los demás participantes, permitiendo ser evaluado, evaluar y autoevaluarse.

Los EVA permiten la construcción conjunta del conocimiento y lenguaje matemático a través de las interacciones entre el facilitador, los participantes y el propio conocimiento matemático programado del aula virtual (Arraiz, 2012).

Lo anterior, permite comprender que las plataformas educativas son herramientas didácticas que contribuyen en los procesos educativos y por ende favorecen la comunicación síncrona y asíncrona, así como también promueven la interacción entre los diferentes actores inmersos en el proceso educativo, razón por la cual resultan ser útiles como herramientas de apoyo para la enseñanza de la Matemática.

Son múltiples los beneficios, pero cabe resaltar que, antes de establecer el EVA a implantar dentro de una institución educativa, es recomendable llevar a cabo un análisis previo de los productos existentes en el mercado, con el

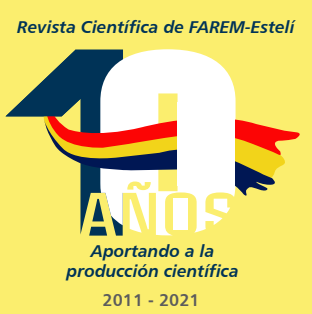


fin de establecer el que se ajusta más a las necesidades particulares, y así crear un marco de trabajo definido y claro a sus actores, quienes han de participar activamente en sus procesos, apoyando las estrategias y metas de la institución (Alarcón et al., 2014)

Por otra parte, estas herramientas virtuales tienen una serie de ventajas, no solo para el docente, sino para el estudiantado que las va a utilizar. Las nuevas tecnologías permiten favorecer el desarrollo de algunas destrezas y habilidades, difíciles de lograr con los medios tradicionales. En concreto, aquellas habilidades que permiten buscar, seleccionar, organizar y manejar nueva información. Favorecen la autonomía en el proceso de aprender, actitudes necesarias para un buen aprendizaje. Mejoran la autoestima, la motivación interna, la disposición a aceptar y comprender múltiples puntos de vista; el respeto por el otro y sus opiniones.

Estas plataformas se caracterizan por tener una interfaz sencilla y amena, lo cual constituye un ambiente estimulante y motivador, de muy fácil navegación, permite la interacción virtual del estudiaste, con el profesor, a través de sus opciones y registros, los cuales no necesariamente, tienen que hacerse en tiempo real, o sea, el estudiante puede acceder a los sitios en cuanto le sea factible, desde y hacia cualquier parte del mundo con solo tener la conectividad adecuada. (Ledo et al., 2008, p. 31)

Los EVA pueden ofrecer importantes resultados en la adquisición de conocimiento matemático del estudiante, mejorando así su rendimiento en esta asignatura considerada de vital importancia por sus aplicaciones inmersas en los distintos ámbitos de la sociedad. Los EVA constituyen un espacio de trabajo que permiten desarrollar el proceso de enseñanzaaprendizaje con implementación de estrategias innovadoras. Un EVA que se ajusta a las necesidades propias de su comunidad educativa, brinda las herramientas necesarias para la creación, desarrollo y puesta en práctica de los cursos y de su respectiva estrategia de enseñanza.

Cabe destacar que el avance del proceso de enseñanza-aprendizaje de la Matemática pasa por una nueva definición de roles, especialmente, para los docentes dado que según (Gómez et al., 2016) en la actualidad, existe la necesidad de cambiar los métodos, técnicas y recursos utilizados por los docentes en el aula, lo cual obliga al profesorado a dejar de ser la única fuente de conocimiento y los EVA, son espacios propicios para este fin, lo cual está de acuerdo con lo afirmado por (González et al., 2018) cuando dicen que una formación virtual centrada en el estudiante y específicamente, en su actividad, obliga a reubicar el rol del docente más justo en la cultura académica.

Cabe mencionar que en Internet se tiene acceso a un amplio número de contenidos relacionados con distintos procesos de enseñanza-aprendizaje de la Matemática, los mismos no necesariamente se ajustan a la estructura planeada para el desarrollo de un curso o las metodologías propuestas por los

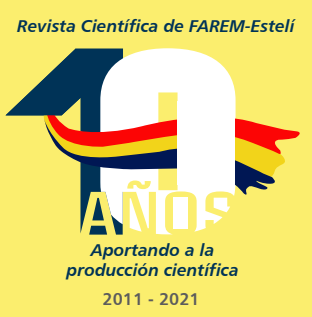


docentes para el abordaje de sus contenidos, de acuerdo con (Mora, 2017), "también debe considerar la calidad y proporcionarles a los estudiantes un recurso coherente con los objetivos de un determinado curso" (p. 107), es así como se debe planear un EVA, de tal manera que responda a las necesidades del curso, a la población a la cual va dirigido y a los objetivos planteados al inicio del mismo.

En relación con la virtualización que de forma paulatina se va dando en algunas instituciones y la madurez que han alcanzado otras. Se debe tener en cuenta que un EVA en el proceso de enseñanza-aprendizaje de la Matemática, implica utilizar materiales digitales, enlaces electrónicos, presentaciones y documentos en distintos formatos, así como el uso de softwares libres. Todos estos recursos, en distintos soportes, son elaborados o recopilados por los docentes y algunas veces son renovados en cada oferta curricular. Entonces, la creación de cursos virtuales representa nuevas oportunidades y también desafíos.

Según Núñez Rodríguez \& Merchor Manaure, (2020), "los EVA permite que el estudiante haga uso de su tiempo fuera de las clases presenciales para la autogestión del conocimiento, con el objeto que el estudiante asimile los contenidos a su ritmo y refuerce lo aprendido en clases" (p. 30). En la asignatura de Matemática, resalta de vital importancia el seguimiento de los docentes mediante la plataforma virtual, porque permite aclarar dudas fuera del horario de clases, necesario cuando se abordan contenidos de mayor complejidad el cual genera muchas dudas.

Los docentes de la asignatura de Matemática pueden considerar entonces un EVA como el espacio que permite desarrollar el proceso pedagógico mediado por las tecnologías. Los ambientes virtuales se convierten en sistemas en los que se encuentran recopilados las didácticas, herramientas y recursos que el docente utiliza con los estudiantes, ya sea de manera virtual o presencial.

Es necesario recalcar que la calidad de un EVA reside no sólo en las herramientas técnicas de las cuales dispone, o en los materiales que incluye, sino en el modo en que se conjugan esas herramientas, materiales y actividades a fin de promover que estudiantes y docentes compartan ideas, conocimientos y destrezas en torno a un saber matemático (Arraiz, 2012). No se debe obviar, que los EVA se desarrollan, a través de la internet y son mediados por una plataforma educativa, por lo que resultan ser estrategias instruccionales que son coordinadas por un tutor con el propósito de facilitar y dinamizar los procesos de enseñanza-aprendizaje.

Respecto al uso de tecnologías, al incorporarlas en la asignatura de Matemática, ofrecen una amplia variedad de recursos y aplicaciones a los docentes. Estas pueden utilizarse como herramientas para realizar cálculos complejos, resolver problemas, dibujar gráficos e interpretar y analizar datos. Esto quiere decir, una ayuda conveniente para el mejoramiento en el proceso

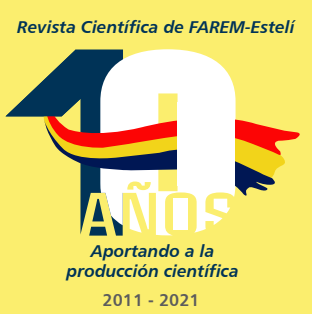


enseñanza-aprendizaje y particularmente de la Matemática, claro está que esto se consigue mediante una aplicación apropiada de la misma, lo cual ha permitido al autor del presente estudio, realizar un análisis referente a los entornos virtuales como recurso para el aprendizaje de la Matemática.

En consecuencia, de todo lo antes descrito, en los resultados presentados por los autores de las distintas investigaciones seleccionadas y analizadas, se logra evidenciar la importancia de los EVA, en el proceso de enseñanza aprendizaje de la Matemática, ya que contribuyen de forma positiva, dando respuestas eficaces a las necesidades del aula y se pueden adaptar en cualquiera de los diferentes niveles de los sistemas educativos, considerándose el uso de la tecnología como apoyo para elevar la calidad educativa.

De acuerdo con estas reflexiones, se coincide que los EVA, favorecen el proceso de enseñanza-aprendizaje de la Matemática. Se pueden considerar como un escenario posible para la construcción de los conocimientos matemáticos, siempre y cuando exista una disposición de los participantes, hacia el fortalecimiento de relaciones de interacción en el sistema didáctico virtual. Con frecuencia y fluidez de la comunicación docente-estudiante se puede garantizar el logro de los objetivos propuestos en el aula virtual.

\section{CONCLUSIONES}

Este estudio de revisión bibliográfica ha permitido precisar varios puntos. En primera medida, el hecho de que la utilización de recursos TIC en el proceso de enseñanza - aprendizaje de la Matemática no puede verse como un sustituto de la labor docente.

La utilización de estos recursos debe verse como una estrategia adicional para lograr, por un lado, motivar al estudiante para la experimentación del concepto a través de simulaciones y herramientas interactivas.

La implementación de EVA permite darle un rol más protagónico al estudiante mismo en la construcción del conocimiento, permitiendo que no sólo sea el docente el dueño absoluto de la información, sino que de manera conjunta estudiante - docente puedan lograr esta construcción.

Los EVA a través de las plataformas promueve la formación integral de los estudiantes y permite la aplicación creativa de sus conocimientos en el desarrollo de habilidades digitales.

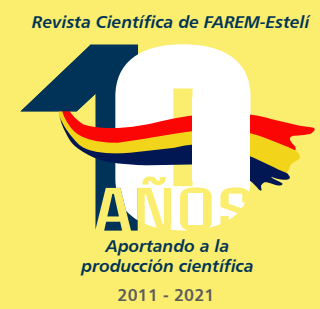


Los EVA contribuyen con nuevas y distintas formas de aprender apoyándose de recursos tecnológicos, que ofreciendo múltiples posibilidades necesarias en un mundo donde prevalece el uso de la tecnología.

En la actualidad es una realidad los beneficios que las tecnologías aportan a las instituciones educativas, es necesario entonces fomentar el uso de las EVA, como oportunidades de mejorar en los procesos de enseñanza-aprendizaje de la Matemática de una mejor manera.

Adecuar las prácticas docentes a las exigencias educativas de hoy en día, fomentando cambio en la visión del educador que quiere lograr sus objetivos de aprendizaje implementando estrategias motivadoras para conseguirlo.

La utilización de recursos tecnológicos conlleva a dar respuestas a las exigencias de la sociedad actual y su uso permite, además, promover la creativa tanto del docente como de los estudiantes a través del aprovechamiento de todo el potencial que ofrecen estos instrumentos tecnológicos.

Una contribución importante en la formación de los estudiantes dada por la tecnología es que los educandos tienen acceso a múltiples recursos de los que se disponen en Internet, los cuales permitirán realizar las adecuaciones curriculares para los alumnos con necesidades educativas especiales.

\section{BIBLIOGRAFÍA}

Alarcón, A. C., Díaz, E. L., \& Callejas-Cuervo, M. (2014). Guía para la evaluación de la usabilidad en los entornos virtuales de aprendizaje (EVA). Información Tecnólogica, 25(3), 135-144. https://doi.org/10.4067/ S0718-07642014000300016

Araque, I., Montilla, L., Meleán, R., \& Arrieta, X. (2018). Virtual Learning Environments: a View From the Theory of Conceptual. Góndola, Enseñanza y Aprendizaje de Las Ciencias, 13(1), 86-100. https://doi. org/10.14483/23464712.11721.

Arraiz, G. (2012). La virtualidad: un escenario posible para la construcción de conocimientos matemáticos. Apertura: Revista de Innovación Educativa, $4(1), 76-85$.

Coca, M. M. (2013). Enseñanza - aprendizaje de las matemáticas en la ESO. Salamanca.

Córdoba, J. (2014). Las TIC en el Aprendizaje de las Matemáticas : ¿ Qué creen los Estudiantes? Congreso Iberoamericano de Ciencia, Tecnología, Innovación y Educación, 1-9. https://doi.org/1571 
Gómez, C., Rodríguez, R., \& Mirete, A. (2016). Percepción de la enseñanza de la historia y concepciones epistemológicas. Una investigación con futuros maestros. Revista Complutense de Educación, 29(1), 237-250. http://dominiodelasciencias.com/ojs/index.php/es/index

González, A. E., Álvarez, G., \& Bassa, L. (2018). Educación virtual en la universidad: un estudio de Investigación-acción para la enseñanza de la asignatura Tecnología educativa. Ciencia, Docencia y Tecnología, 29(57), 201-234. https://doi.org/10.33255/2957/357

Gutiérrez Rodríguez, C. A. (2018). Fortalecimiento de las competencias de interpretación y solución de problemas mediante un entorno virtual de aprendizaje. Enero - Junio, 8(2), 279-293. https://doi. org/10.19053/20278306.v8.n2.2018.7170

Ledo, M., Ruiz, S., Oliete, F., \& Vidal, N. (2008). Entornos virtuales de enseñanza-aprendizaje Teaching-learning virtual settings. Educación Médica Superior, 22(1), 1-9.

Mora, F. (2017). Objetos de aprendizaje: importancia de su uso en la educación virtual Revista Calidad en la Educación Superior Programa de Autoevaluación Académica Universidad Estatal a Distancia ISSN 16594703 EDUCACIÓN VIRTUAL LEARNING OBJECTS : THE IMPORTANCE OF IT '. Dial, 3(January 2012), 15. https://www.researchgate.net/ publication/277270560_Objetos_de_aprendizaje_importancia_de_su_ uso_en_la_educacion_virtual

Núñez Rodríguez, J. A., \& Merchor Manaure, G. A. (2020). Implementación de un entorno virtual en el proceso de enseñanza-aprendizaje-investigación de la asignatura "Histotecnología III." Revista Científica de FAREM-Estelí, 32, 24-33. https://doi.org/10.5377/farem.v0i32.9227

Pacheco, L., Díaz, E., Ávila, W., \& Rodríguez, V. (2017). Las Tics en los procesos de enseñanza y aprendizaje en la educación universitaria. Las Tics en los Procesos de Enseñanza y Aprendizaje En la Educación Universitaria, 3(2), 721-749.

Pineda, V. O., \& Leyva, J. T. (2013). Entornos Virtuales de Enseñanza yAprendizaje: La Educación entre lo Presencial y lo Virtual. Entornos Virtuales de Enseñanza y Aprendizaje: La Educación Entre lo Presencial y lo Virtual, 10(2), 435-442. https://doi.org/10.14483/2322939X.6567

Pino, S., \& Salazar, Y. (2018). Afianzando el aprendizaje de las matemáticas a través de una EVA orientado a fortalecer el pesamineto métrico y los sistemas de medidas en el primer ciclo de la básica primaria. Universidad libre.

Revelo, J., \& Carrillo, S. (2018). Impacto del uso de las TIC como herramientas para el aprendizaje de la matemática de los estudiantes de educación media. Cátedra, 1(1), 70-91. https://doi.org/10.29166/catedra. v1i1.764

Rodríguez, J., Romero, J., \& Vergara, G. (2017). Importancia de las TIC en enseñanzas de las matemáticas. Revista MATVA, 2, 41-49.

Rugama, J. A. G. (2017). Proceso de enseñanza-aprendizaje de la integral

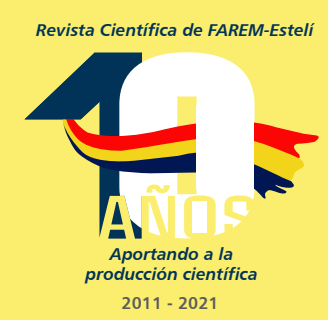


definida como el área bajo una curva en las asignaturas de Cálculo en las carreras de Ingeniería.

Scharfstein, M., \& Gaurf. (2013). Comunicación y educación en entornos virtuales de aprendizaje. Perspectivas teóricos-metodológicas. Journal of Chemical Information and Modeling, 53(9), 1689-1699. https://doi. org/10.1017/CBO9781107415324.004 